'Unidad de Hematología

Intensiva y trasplante de

progenitores hematopoyéticos,

Hospital del Salvador, Servicio

de Salud Metropolitano Oriente, Santiago de Chile.

${ }^{2}$ Escuela de Kinesiología.

Universidad Andrés Bello. Santiago, Chile.

${ }^{3}$ Programa Nacional de Trasplante de progenitores hematopoyéticos de adultos, Ministerio de Salud. aKinesiólogo. ${ }^{\mathrm{b}} \mathrm{PhD}$.

Este trabajo no ha recibido apoyo financiero.

Recibido el 19 de junio de 2015, aceptado el 24 de junio de 2016.

Correspondencia a: Luz Alejandra Lorca Unidad de Hematología Intensiva, Hospital del Salvador, Avenida Salvador 364, Providencia, Santiago de Chile. alejandralorcap@gmail.com

\section{Propiedades psicométricas del inventario breve de fatiga en personas tratadas por neoplasias hematológicas en Chile}

\author{
LUZ ALEJANDRA LORCA ${ }^{1, a}$, CINARA SACOMORI ${ }^{2, a, b}$, \\ BÁRBARA PUGA ${ }^{1,3}$
}

\section{Assessment of a brief fatigue inventory in patients with hematologic malignancies}

Background: Fatigue is one of the most common and distressing symptoms experienced by cancer patients. Aim: To validate the Brief Fatigue Inventory in people treated for hematological neoplasms. Material and Methods: In a cross-sectional study, the Brief Fatigue Inventory was answered by 122 patients aged $40 \pm 14$ years (50\% women) treated for hematological neoplasms at an intensive hematological unit of a public hospital between July 2010 and July 2013. Socio-demographic and clinical parameters were obtained from their clinical records. Results: Fatigue was present in nearly all patients (99.2\%) in minor (50\%), moderate (36.9\%) or severe (12.3\%) levels. The average fatigue score was $4.5 \pm 1.9)$. The Brief Fatigue Inventory had a good internal consistency (Cronbach's alpha $=0.973$ ) and proved to be one-dimensional $(84.3 \%$ of the explained variance). Women reported that fatigue interfered more with enjoyment of life than men $(p=0.036)$. Conclusions: The Brief Fatigue Inventory is a reliable instrument that can be used in clinical practice. It allows a quick assessment of the level of fatigue. People treated for hematologic cancer have a high prevalence of fatigue.

(Rev Med Chile 2016; 144: 894-899)

Key words: Fatigue; Hematologic Neoplasms; validation Studies.

\footnotetext{
L
} a fatiga relacionada al cáncer se define como una sensación preocupante, persistente y subjetiva de cansancio o agotamiento físico, emocional o cognitivo, que no es proporcional a la actividad realizada ${ }^{1}$. La presencia de fatiga se asocia a trastornos psicológicos, sintomáticos y disminución del estado funcional ${ }^{2}$ y puede ser causada por la enfermedad en sí, o por los diversos tratamientos como la quimioterapia, la radioterapia, terapia biológica o cirugía ${ }^{3}$. Tiende a aumentar con el curso del tratamiento y puede persistir por años, aunque para la mayoría se disipa después del tratamiento ${ }^{4}$. Además, existen otros mecanismos causantes de fatiga en pacientes oncológicos, como los trastornos de sueño, las condiciones ambientales, el nivel de actividad y el estado nutricional ${ }^{5}$.

La fatiga es uno de los efectos a largo plazo más prevalentes y preocupantes del tratamiento contra el cáncer, afectando de manera significativa el funcionamiento y la calidad de vida de los pacientes ${ }^{3}$. Su prevalencia ha sido descrita entre 59\% y $100 \%$, dependiendo del estado clínico de la enfermedad ${ }^{5}$.

Los pacientes tratados por neoplasias hematológicas, particularmente, pueden presentar niveles elevados de fatiga en el ambiente hospitalario, en parte, a causa de la quimioterapia o el trasplante de progenitores hematopoyéticos, los cuales corresponden a tratamientos agresivos ${ }^{6}$. 
Entre los instrumentos específicos para evaluar fatiga en la población con cáncer, se destaca el Inventario Breve de Fatiga (IBF) por ser un instrumento ampliamente utilizado, corto, de fácil administración y calificación ${ }^{3}$. Está disponible en idioma español, pero no estaba validado para la población oncológica de Chile. Por tanto, los objetivos de este estudio fueron: validar el Inventario Breve de Fatiga en personas tratadas por neoplasias hematológicas en Chile, describir la fatiga oncológica en ambiente hospitalario y comparar el nivel de fatiga entre hombres y mujeres.

\section{Material y Método}

Corresponde a un estudio transversal de validación que utilizó información de las fichas clínicas, específicamente las evaluaciones realizadas por un kinesiólogo.

\section{Participantes}

Los participantes de este estudio fueron pacientes tratados por neoplasias hematológicas en la Unidad de Hematología Intensiva del Hospital del Salvador entre julio de 2010 y julio de 2013, los que fueron evaluados por el kinesiólogo asistencial de la Unidad. Éste evaluaba de forma rutinaria, en sus horas clínicas, a todos los pacientes que egresaban de la unidad. El entrevistador, uno de los autores de este estudio, fue entrenado para leer las preguntas y no interferir en las respuestas de los usuarios.

Los criterios de inclusión para entrar al estudio fueron: paciente con diagnóstico de neoplasias oncológicas que ingresa para tratamiento a la Unidad de Hematología Intensiva del Hospital del Salvador y edad mayor a 18 años. Como criterio de exclusión se consideró a aquellos pacientes cuya ficha clínica no contaba con la evaluación de fatiga o no estaba completa. La evaluación kinésica de la fatiga no era realizada en aquellos pacientes que estaban inconscientes, no colaborativos o fueron derivados a la Unidad de Cuidados Paliativos.

El tamaño de la muestra fue calculado en función del análisis principal del estudio, análisis factorial, puesto que se recomienda un mínimo de 10 personas para cada ítem del cuestionario ${ }^{7}$.

La muestra quedó constituida por 122 pacientes, 61 hombres (50\%) y 61 Mujeres (50\%).
La Tabla 1 presenta la caracterización de los participantes respecto a edad, sexo, diagnóstico del paciente y tipo de tratamiento oncológico (quimioterapia intensiva, trasplante autólogo o alogénico de progenitores hematopoyéticos de sangre periférica $[\mathrm{TPH}]$ ). Cabe señalar que no se consideró las comorbilidades, los síntomas (vómitos, diarrea, otros), el estadio del cáncer ni los días de hospitalización.

El estudio fue aprobado por el Comité de Ética Científico del Servicio de Salud Metropolitano Oriente (Carta $N^{\circ}$ 012/UHI con fecha 01 de julio de 2014), el cual otorgó el correspondiente permiso para tomar los datos de la ficha clínica sin necesidad de consentimiento informado, considerando que la toma de datos empezó antes de 2012, año de la aprobación del reglamento para la constitución y funcionamiento de los Comités de Ética Asistencial. De todos modos, durante todo el proceso fueron considerados los criterios éticos de la declaración de Helsinki y se respetó la confidencialidad de los datos.

Tabla 1. Características demográficas, diagnósticos y trasplantados según donante de la población en estudio $(n=122)$

\begin{tabular}{|lrr|}
\hline Característica & $\mathbf{n}$ & $\mathbf{( \% )}$ \\
\hline Sexo & & \\
$\quad$ Masculino & 61 & $(50)$ \\
Femenino & 61 & $(50)$ \\
Edad & & \\
$15-24$ & 32 & $(26,3)$ \\
$25-34$ & 22 & $(18,0)$ \\
$35-44$ & 23 & $(18,8)$ \\
$45-54$ & 32 & $(26,3)$ \\
$55-64$ & 13 & $(10,6)$ \\
Diagnóstico & & \\
Leucemia linfoblástica aguda & 28 & $(23,0)$ \\
Leucemia mieloide aguda & 34 & $(27,9)$ \\
Leucemia promielocítica aguda & 14 & $(11,5)$ \\
Linfoma Hodgkin & 17 & $(13,9)$ \\
Linfoma no Hodgkin & 3 & $(2,5)$ \\
Mieloma múltiple & 11 & $(9,0)$ \\
Linfoma Burkitt & 5 & $(4,1)$ \\
Otros & 10 & $(8,2)$ \\
Trasplantados según donante & & \\
Trasplante alogénico & 8 & $(6,5)$ \\
Trasplante autólogo & 32 & $(26,2)$ \\
\hline
\end{tabular}




\section{Instrumento}

\section{Inventario breve de fatiga (IBF)}

El IBF fue desarrollado y validado en Estados Unidos de Norteamérica en relación a validez de constructo, concurrente y discriminante ${ }^{3}$. Además, demostró ser unidimensional y presentó buena consistencia interna con alfa de Cronbach de $0,96^{3}$. Presentó buena confiabilidad test-retest en Alemania ${ }^{8}$ y en Taiwan ${ }^{9}$. Los autores de la versión original dispusieron la versión validada en español del instrumento y dieron el permiso para su uso en este estudio ${ }^{10}$.

El instrumento consta de 9 ítems en escala de 0 a 10. Tres ítems evalúan la severidad de la fatiga (peor fatiga, fatiga usual y fatiga en el momento actual), donde el 0 se refiere a ninguna fatiga $y$ el 10 a la peor fatiga que se puede imaginar. Los otros seis ítems evalúan la forma en que la fatiga ha interferido en distintos aspectos de la vida (actividad en general, estado de ánimo, capacidad para caminar, trabajo normal, relaciones con otras personas y capacidad de diversión). Para esos últimos 0 equivale a no interfiere y 10 interfiere por completo. El punto de corte está dada por el ítem 3 (Por favor, califique su fatiga (cansancio) haciendo un círculo alrededor del número que describe la PEOR fatiga durante las últimas $24 \mathrm{~h}$ ) y categoriza a los pacientes según puntuación en donde el 0 significa: "sin fatiga"; 1-3: "leve"; 4-6: "moderada" y 7-10: "severa" 3 . Es posible llegar a un puntaje de fatiga haciendo un promedio aritmético de todos los ítems, mientras mayor el valor peor la fatiga.
En ese estudio, el instrumento fue aplicado en formato de entrevista por un único evaluador.

\section{Análisis de datos}

Los datos fueron analizados usando el programa estadístico SPSS con recursos de estadística descriptiva (frecuencia absoluta y relativa, media, mediana y desviación estándar) e inferencial. Se realizó la prueba de normalidad de Kolmogorv Smirnov y, como la fatiga no presentó distribución normal, se utilizó el test no paramétrico U de Mann Whitney para comparación de la fatiga entre hombres y mujeres. Para evaluar la consistencia interna del instrumento (confiabilidad interna) se utilizó el análisis de alfa de Cronbach. Por último, se realizó un análisis factorial exploratorio de los componentes principales con método de rotación Varimax. Para todos los casos se estableció un nivel de significación de $\alpha=0,05$.

\section{Resultados}

La media de edad de los participantes fue 39,93 ( $\mathrm{DS}=13,68)$. La mayoría de ellos tenía diagnóstico de leucemia aguda y $32,7 \%$ fueron sometidos a trasplante de progenitores hematopoyéticos (Tabla 1).

La fatiga estuvo presente en casi todos los pacientes $(99,2 \%, \mathrm{n}=121)$, en niveles leve $(50 \%$, $\mathrm{n}=61)$, moderada $(36,9 \%, \mathrm{n}=45)$ o severa $(12,3 \%$, $\mathrm{n}=15)$. La media del puntaje de los ítems de la fatiga fue 4,51 (DS =1,89). Los datos descriptivos para cada ítem de la escala de fatiga están presentados en la Tabla 2. El ítem que presentó mayor media, peor

Tabla 2. Fatiga de los participantes con cánceres hematológicos al momento del alta hospitalario $(\mathbf{n}=122)$

\begin{tabular}{|c|c|}
\hline Ítems del inventario breve de fatiga & X (DS) \\
\hline 1. Fatiga en este momento & $4,53(2,08)$ \\
\hline 2. Fatiga usual durante las últimas $24 \mathrm{~h}$ & $4,52(2,09)$ \\
\hline 3. Peor fatiga durante las últimas $24 \mathrm{~h}$ & $4,55(2,08)$ \\
\hline 4. Cuánto su fatiga interfiere en su actividad en general durante las últimas $24 \mathrm{~h}$ & $4,54(2,09)$ \\
\hline 5. Cuánto su fatiga interfiere en su estado de ánimo durante las últimas 24 h & $4,48(2,00)$ \\
\hline 6. Cuánto su fatiga interfiere en su capacidad para caminar durante las últimas $24 \mathrm{~h}$ & $4,38(2,00)$ \\
\hline 7. Cuánto su fatiga interfiere en su trabajo normal (ya sea en su casa o afuera del hogar) durante las últimas $24 \mathrm{~h}$ & $3,22(1,53)$ \\
\hline 8. Cuánto su fatiga interfiere en sus relaciones con otras personas durante las últimas $24 \mathrm{~h}$ & $4,71(2,30)$ \\
\hline 9. Cuánto su fatiga interfiere en su capacidad de diversión (disfrutar la vida) durante las últimas 24 h & $5,67(2,53)$ \\
\hline Puntaje de fatiga & $4,51(1,89)$ \\
\hline
\end{tabular}

$\mathrm{X}=$ media; $\mathrm{DS}=$ desviación estándar. 
Tabla 3. Análisis factorial del inventario breve sobre la fatiga

\begin{tabular}{|lc|}
\hline Ítems & $\begin{array}{c}\text { Carga } \\
\text { factorial }\end{array}$ \\
\hline 1. Fatiga en este momento & 0,985 \\
\hline 2. Fatiga usual durante las últimas $24 \mathrm{~h}$ & 0,988 \\
\hline 3. Peor fatiga durante las últimas $24 \mathrm{~h}$ & 0,988 \\
\hline 4. Cuánto su fatiga interfiere en su actividad en general durante las últimas 24 h & 0,984 \\
\hline 5. Cuánto su fatiga interfiere en su estado de ánimo durante las últimas 24 h & 0,981 \\
\hline 6. Cuánto su fatiga interfiere en su capacidad para caminar durante las últimas 24 h & 0,968 \\
\hline 7. Cuánto su fatiga interfiere en su trabajo normal (ya sea en su casa o afuera del hogar) durante las últimas 24 h & 0,665 \\
\hline 8. Cuánto su fatiga interfiere en sus relaciones con otras personas durante las últimas 24 h & 0,913 \\
\hline 9. Cuánto su fatiga interfiere en su capacidad de diversión (disfrutar la vida) durante las últimas $24 \mathrm{~h}$ & 0,722 \\
\hline
\end{tabular}

*El análisis cumplió con los presupuestos: $\mathrm{KMO}=0,922$, test de esfericidad de Bartlett presentó $\mathrm{p}<0,001$.

fatiga, es el que medía el impacto que tiene la fatiga en la capacidad de disfrutar la vida; mientras que el ítem con menor media fue el relativo a cuánto la fatiga afectaba en su trabajo normal.

En cuanto a las propiedades psicométricas del IBF, se observó que éste presentó buena consistencia interna (alfa de Cronbach $=0,973$ ). El análisis factorial, presentado en la Tabla 3 , generó un único factor responsable por $84,34 \%$ de la varianza explicada. Los ítems que tuvieron menores valores de carga factorial fueron los relativos a capacidad para el trabajo y poder disfrutar la vida.

El nivel de fatiga fue comparado entre hombres y mujeres participantes de este estudio. Se observó que hubo diferencia significativa entre hombres y mujeres solamente en relación a la variable "cuánto la fatiga interfiere en el poder disfrutar la vida" $(\mathrm{p}=0,036)$; de modo que las mujeres (Media $=$ $6,1 ; \mathrm{DS}=2,5$; Mediana $=7,0)$ reportaron que la fatiga las limitaba más en poder disfrutar de la vida que los hombres (Media $=5,2 ; \mathrm{DS}=2,5$; Mediana $=5,0)$.

\section{Discusión}

En este estudio fueron evaluadas las propiedades psicométricas del Inventario Breve de Fatiga y observado que este presentó buen nivel de confiabilidad interna y demostró ser unidimensional. El IBF demostró buen nivel de consistencia interna $(\alpha=0,973)$. La consistencia interna es una medida de confiabilidad interna del instrumento que da soporte a la validez del instrumento. Se describe que un valor de alfa superior a 0,8 es lo deseado ${ }^{7}$. De ese modo, el valor del alfa obtenido indica que todas las preguntas del IBF miden el mismo constructo, la fatiga. Ese valor fue similar al encontrado en otros estudios con pacientes con distintos tipos de cánceres ${ }^{3,11,12}$. Es posible que la menor carga factorial obtenida en los ítems referentes a trabajo y poder disfrutar la vida sea debido a la condición de hospitalizado, a la vez que en ese ambiente estas actividades están muy limitadas.

En nuestro estudio, el IBF demostró ser unidimensional, del mismo modo que otros estudios con población con neoplasias en Estados Unidos de Norteamérica ${ }^{3}$, Grecia $^{11}$, Japón ${ }^{13}$, Taiwan ${ }^{9}$, Korea $^{12}$ e Italia ${ }^{14}$. Los autores nombran esa dimensión como "severidad de fatiga" ${ }^{3}$. Mientras tanto, un estudio de validación del instrumento en una población de adultos mayores de la comunidad en Estados Unidos de Norteamérica ${ }^{13}$ y otro estudio de validación del instrumento en pacientes con cáncer ${ }^{15}$ identificó dos factores: severidad de la fatiga e interferencia. Es posible que la condición de hospitalizado haya interferido en el resultado de ese estudio. La restricción a la cama hospitalaria pudo haber contribuido para que la severidad de la fatiga y la interferencia de la fatiga no fueran diferenciadas por los pacientes.

Los otros instrumentos más utilizados para evaluar la fatiga relacionada al cáncer se proponen a evaluar más dimensiones de la fatiga. El Inventario Multidimensional de Fatiga considera 
cinco dimensiones: fatiga general, fatiga física, fatiga mental, actividad reducida y motivación reducida ${ }^{16}$. La escala de Piper propone cuatro dimensiones: comportamental/severidad, significado afectivo, sensorial y cognitivo/humor ${ }^{17}$. Además, algunos instrumentos de calidad de vida en población con cáncer contemplan la dimensión fatiga, como el cuestionario de la Organización Europea para Investigación y Tratamiento del Cáncer, el Quality of Life Questionnaire Core $30^{1}$. Comparando con los instrumentos anteriormente mencionados, el IBF se destaca por ser breve y de fácil aplicación. Otra ventaja del uso del IBF es que ha sido validado en muchos países del mundo, lo que facilita la comparación de los datos y la síntesis de conocimiento sobre la fatiga relacionada al cáncer.

Este estudio verificó alta prevalencia de fatiga relacionada al cáncer al momento de egreso hospitalario en personas tratadas por neoplasias hematológicas con quimioterapia intensiva y/o trasplante de progenitores hematopoyéticos, siendo que $49,2 \%$ de los participantes de este estudio reportaron sentir fatiga severa o moderada. Similares hallazgos han obtenido en pacientes con otros tipos de cáncer reportándose fatiga modera o severa en $48 \%{ }^{13,15}$.

Se observó que el ítem relativo al impacto que tenía la fatiga en la capacidad de disfrutar la vida obtuvo el mayor puntaje. Ese hallazgo refuerza la necesidad de adoptar medidas para prevenir y minimizar la fatiga, objetivando mejorar el bienestar de las personas tratadas por neoplasias hematológicas durante el período de tratamiento en el cual permanecen hospitalizados. Para eso, algunos estudios han sugerido que los ejercicios físicos $^{18}$, la músico-terapia cognitivo-comportamental ${ }^{19} \mathrm{y}$ la meditación reducen la fatiga durante los tratamientos ${ }^{20}$. No obstante, entre las terapias anteriormente mencionadas, la que presenta buen nivel de evidencia es el ejercicio físico ${ }^{18}$.

Por último, fue observado que las mujeres de este estudio reportaron fatiga con mayor limitación para disfrutar de la vida que los hombres. Otros estudios con pacientes oncológicos sometidos a TPH, también resultaron que las mujeres tenían mayor fatiga que los hombres ${ }^{21,22}$, principalmente la fatiga física ${ }^{21}$. Además, fue demostrado que en adultos mayores con distintos tipos de cáncer, las mujeres presentaban más fatiga que los hombres ${ }^{23}$. Por otro lado, otro estudio que evaluó las diferencias de género en relación a la calidad de vida de pacientes con dolor asociado a cáncer, no identificó diferencia entre hombres $y$ mujeres en cuanto a fatiga ${ }^{24}$. Algunos autores atribuyen esa diferencia a factores biológicos y psicológicos ${ }^{21}$, pero también es posible que el rol social de las mujeres, que suele incluir más tareas domésticas y de soporte a los otros miembros de la familia, explique por qué la fatiga las limitaba más en disfrutar la vida.

\section{Conclusiones}

El Inventario Breve de Fatiga es un instrumento válido y confiable con gran potencial de utilidad en la práctica clínica, puesto que permite una evaluación rápida del nivel de fatiga en pacientes tratados por neoplasias hematológicas e identifica aquellos pacientes con fatiga moderada o severa, los cuales deberían ser tratados.

Además, este estudio identificó elevada prevalencia de fatiga en esos pacientes y que, según las mujeres de este estudio, la fatiga las limitaba más en disfrutar la vida comparado con los hombres. Estos resultados permiten sugerir que pacientes tratados por cáncer debieran contar rutinariamente con una evaluación de fatiga y que recursos de salud debieran ser orientados a su rehabilitación, favoreciendo la gestión del cuidado continuo de esta población y aportando en su reinserción social y laboral.

Este estudio presenta como limitaciones no haber considerado las comorbilidades, los síntomas (vómitos, diarrea, otros), el estadio del cáncer y los días de hospitalización. Sin embargo, presenta como fortaleza haber sido realizado en el centro de referencia nacional para trasplante de progenitores hematopoyéticos y centro de derivación nacional para leucemias y linfomas agresivos. Así, es probable que sea el primer estudio en nuestro país que evaluó la fatiga en pacientes tratados por neoplasias hematológicas, reportando datos psicométricos del IBF.

Agradecimientos: Agradecemos a los autores del IBF por facilitar la versión en español. Se recomienda para su utilización en nuestro país de este instrumento, contar con la autorización de los autores de la versión original en el sitio web (http://www3.mdanderson.org/depts/symptomresearch/). 


\section{Referencias}

1. Bower JE, Bak K, Berger A, Breitbar W, Escalante CP, Ganz PA, et al. Screening, assessment, and management of fatigue in adult survivors of cancer: An American Society of Clinical Oncology clinical practice guideline adaptation. J Clin Oncol 2014; 32 (17): 1840-51.

2. Irvine D, Vincent L, Graydon JE, Bubela N, Thomson L. The prevalence and correlates of fatigue in patients receiving treatment with chemotherapy and radiotherapy: A comparison with the fatigue experienced by healthy individuals. Cancer Nurs 1994; 17 (5): 367-78.

3. Mendoza TR, Wang XS, Cleeland CS, Morrissey M, Johnson BA, Wendt JK, et al. The rapid assessment of fatigue severity in cancer patients. Cancer 1999; 85: 1186-96.

4. Fitch MI, Buston T, Bakker D, Mings D, Sevean P. The Fatigue Pictogram: Psychometric evaluation of a new clinical tool. Can Oncol Nurs J 2011; 21 (4): 205-17.

5. Yeh C-T, Wang L-S. Potential pathophysiological mechanism of cancer-related fatigue and current management. Formos J Surg 2014; 47 (5): 173-82.

6. Prieto JM, Blanch J, Atala J, Carreras E, Rovira M, Cirera $\mathrm{E}$, et al. Clinical factors associated with fatigue in hematologic cancer patients receiving stem-cell transplantation. Eur J Cancer 2006; 42 (12): 1749-55.

7. Field A. Descobrindo a estatística usando o SPSS. 2. ed. Porto Alegre: Artmed, 2009. P.570.

8. Radbruch L, Sabatowski R, Elsner F, Everts J, Mendoza T, Cleeland C. Validation of the German version of the Brief Fatigue Inventory. Journal of Pain and Symptom Management 2003; 25 (5): 449-57. J Pain Symptom Manage 2003; 25 (5): 449-57.

9. Lin CC, Chang AP, Chen ML, Cleeland CS, Mendoza TR, Wang XS. Validation of the Taiwanese version of the Brief Fatigue Inventory. J Pain Symptom Manage 2006; 32 (1): 52-9.

10. Valenzuela JO, Gning I, Irarrázaval ME, Fasce G, Marín L, Mendoza TR, et al. Psychometric validation of the Spanish version of the Brief Fatigue Inventory [abstract]. The University of Texas MD Anderson Cancer Center, Division of Internal Medicine Research Retreat, Houston TX, May 24, 2012.

11. Mystakidou K, Tsilika E, Parpa E, Mendoza TR, Pistevou-Gombaki K, Vlahos L, et al. Psychometric properties of the Brief Fatigue Inventory in Greek patients with advanced cancer. J Pain Symptom Manage 2008; 36 (4): 367-73.

12. Yun YH, Wang XS, Lee JS, Roh JW, Lee CG, Lee WS, et al. Validation study of the Korean version of the Brief Fatigue Inventory. J Pain Symptom Manage 2005; 29
(2): 165-72.

13. Okuyama T, Wang XS, Akechi T, Mendoza TR, Hosaka T, Cleeland CS, et al. Validation study of the Japanese version of the Brief Fatigue Inventory. J Pain Symptom Manage 2003; 25 (2): 106-17.

14. Catania G, Bell C, Ottonelli S, Marchetti M, Bryce J, Grossi A, et al. Cancer-related fatigue in Italian cancer patients: validation of the Italian version of the Brief Fatigue Inventory (BFI). Support Care Cancer 2013; 21 (2): 413-9.

15. Wang XS, Hao XS, Wang Y, Guo H, Jiang YQ, Mendoza RT, et al. Validation study of the Chinese version of the Brief Fatigue Inventory (BFI-C). J Pain Symptom Manage 2004; 24 (4): 322-32.

16. Smets EMA, Garssen B, Bonke B, De Haes JCJM. The Multidimensional Fatigue Inventory (MFI) psychometric qualities of an instrument to assess fatigue. J Psychosom Res 1995; 39: 315-25.

17. Dagnelie PC, Pijls-Johannesma MCG, Pijpe A, Boumans BJE, Skarabanja ATP, Lambin P, et al. Phsychometric properties of the revised Piper Fatigue Scale in Dutch cancer patients were satisfactory. J Clin Epidemiol 2006; 59: 642-9.

18. Persoon S, Kersten MJ, Van der Weiden K, Buffart LM, Nollet F, Brug J, et al. Effects of exercise in patients treated with stem cell transplantation for a hematologic malignancy: a systematic review and meta-analysis. Cancer Treat Rev 2013; 39 (6): 682-90.

19. Fredenburg HA, Silverman MJ. Effects of cognitive-behavioral music therapy on fatigue in patients in a blood and marrow transplantation unit: A mixed-method pilot study. The Arts in Psychotherapy 2014; 41: 433-44.

20. Kim YH, Kim HJ, Ahn SD, Seo YJ, Kim SH. Effects of meditation on anxiety, depression, fatigue, and quality of life of women undergoing radiation therapy for breast cancer. Complementary Ther Med 2013; 21: 379-87.

21. Knobel H, Loge JH, Nordøy T, Kolstad AL, Espevik T, Kvaløy S, et al. High level of fatigue in lymphoma patients treated with high dose therapy. J Pain Symptom Manage 2000; 19 (6): 446-56.

22. Cohen MZ, Rozmus CL, Mendoza TR, Padhye NS, Neumann J, Gning I, et al. Symptoms and quality of life in diverse patients undergoing hematopoietic stem cell Lantation. J Pain Symptom Manage 2012; 44 (2): 168-80.

23. Respini D, Jacobsen PB, Thors C, Tralongo P, Balducci L. The prevalence and correlates of fatigue in older cancer patients. Crit Rev Oncol Hematol 2003; 47 (3): 273-9.

24. Pud D. Gender differences in predicting quality of life in cancer patients with pain. Eur J Oncol Nurs 2011; 15 (5): 486-91. 